\title{
Le chroniqueur n'aime pas les lecteurs - ni les journalistes : Henri Roorda et la presse
}

\author{
Anne-Lise Delacrétaz \\ Université de Lausanne \\ ORCID: 0000-0003-0219-8519
}

\begin{abstract}
Entre 1917 et 1925, Henri Roorda publie sous le pseudonyme de Balthasar près de six cent cinquante chroniques dans les quotidiens suisses romands, dont quelques-unes consacrées aux journaux et aux journalistes. Comment Roorda compose-t-il avec les changements qui transforment profondément la presse au tournant du $\mathrm{Xx}^{\mathrm{e}}$ siècle ? Il dénonce l'autorité du journaliste, que ce dernier en use ou pas, et la docilité du lecteur, le remplissage des journaux, la manipulation de l'opinion. Contre l'information de masse, dont il pressent les dangers, il adopte la posture ambiguë de l'humoriste et fait un plaidoyer pour la « diversité dans l'unité ».
\end{abstract}

Keywords : Henri Roorda, chronique, presse romande, Tribune de Lausanne, Gazette de Lausanne, Tribune de Genève.

Maître de mathématiques à Lausanne - dès I892 à l'École supérieure de jeunes filles et dès I905 au Collège et au Gymnase classique cantonal -, Henri Roorda van Eysinga se lance tardivement dans une courte et intense carrière de chroniqueur, « dont l'art consiste à effleurer les questions, à improviser une causerie aussi ingénieuse et intéressante que possible sur n'importe quel sujet », selon la définition d'un contemporain, Alexis de Chambure (de Chambure 19I4, cité par Thérenty $2007: 236$ ). Durant les dix dernières années de sa vie, il donne en effet près de six cent cinquante chroniques dans la presse romande - pour nous en tenir à sa production régulière ${ }^{1}$.

Comment Roorda décroche-t-il, en pleine guerre, une collaboration à la Tribune de Lausanne, lui qui n'a publié, à défaut du roman anarchiste projeté, que quelques articles notamment sur la pédagogie dans la Gazette de Lausanne ou dans des revues anarchistes et libertaires françaises peu connues du public vaudois comme Les Temps nouveaux, L'Humanité nouvelle et La Revue blanche ? À l'époque, les chroniques sont généralement tenues par des écrivains - souvent en mal de copie ou d'argent. Entre I9I3 et I9I8, C. F. Ramuz, par exemple, romancier déjà affirmé, livre une centaine de courts textes réflexifs «À propos de tout » dans la Gazette de Lausanne, dont il partage sporadiquement le sommaire du dimanche avec Roorda.

I Un choix de ses chroniques est publié dans Roorda 2013, dans Roorda 2020 et dans Roorda 202I. Pour le détail de ses collaborations, voir Michel Froidevaux, « Note sur les textes proposés» (Roorda 2009: IO). 
Sans doute est-ce la parution, en I917, du Pédagogue n'aime pas les enfants dans les Cahiers vaudois, où il a été introduit par son collègue et ami Edmond Gilliard $^{2}$, plutôt que celle de ses livres d'arithmétique et de géométrie aux Éditions Payot, qui assied la notoriété de Roorda jusqu'alors relativement discrète, comme le confirme un article de la Gazette de Lausanne du 22 juin 1913 - signé du musicologue Ernest Ansermet, lui aussi maître de mathématiques au Collège classique cantonal :

Connu du public indirectement surtout, et par la réputation que lui a value une activité pédagogique remarquable, $M$. Roorda van Eysinga n'avait guère communiqué directement avec lui - exception faite de quelques articles épars dans les revues -, que par des conférences d'ailleurs très remarquées. Les ouvrages qu'il est en train de publier, outre le service qu'ils vont rendre aux élèves de nos écoles secondaires, permettront de le mieux connaître et d'apprécier en lui le constructeur ou le créateur alors que jusqu'ici on a vu surtout le critique.

De I9I7 à 1919, Roorda publie chaque semaine dans la Tribune de Lausanne des chroniques dont le titre général, « La vie ordinaire », pour lui avoir été imposé par le journal, ne lui permet pas moins d'affirmer à mots couverts son horreur de la guerre et son aspiration à la routine : « N'ayant aucun goût pour la vie héroïque et les actions d'éclat, j’ai été chargé par la Direction de la Tribune de Lausanne d'entretenir mes lecteurs de choses de la vie ordinaire» (« La fin des guerres », La Tribune de Lausanne, 24 juin 1917)

En I919, il passe à la Gazette de Lausanne " qui paie mieux », avoue-t-il le II février 1920 à son ami Jacques Gross, proche comme lui des cercles anarchistes ${ }^{4}$; d'abord irrégulières, ses chroniques, intitulées "Ne nous frappons pas », deviennent hebdomadaires de 1920 à 1924, puis bimensuelles de 1924 jusqu'à sa mort en 1925. À partir de I923, Roorda s'illustre aussi dans la Tribune de Genève qui lui ouvre sa rubrique «Au fil des jours »".

S'il réserve son patronyme complet à ses articles sérieux, Roorda signe ses contributions légères du pseudonyme de Balthasar, donnant à son double humoristique le nom du grand roi mythique de Babylone, dont il se présente comme le dernier descendant, non sans une touche d'autodérision, de mégalomanie et de prudence - la dissimulation ou plutôt la fictionnali-

\footnotetext{
2 Voir Carine Corajoud, "Gilliard et Roorda : deux expressions pour une même voix " (Enckell et al. 2009 : 93-IO2).

3 Cette chronique est reprise sous le même titre mais sans cet incipit dans Roorda (2012 : I4-I7).

4 Cité par Gilles Losseroy, « Roorda, anarchistes de père en fils » (Enckell et al. 2009 : 57).

5 Entre les années 1922 et 1925, Roorda publie quatre Almanachs Balthasar aux Éditions Spes, «support d'expression personnel » où il peut «à sa guise choisir la matière, les images, la mise en page pour laisser libre cours à sa fantaisie et à sa verve " (Michel Froidevaux, "Les Almanachs de Balthasar », Enckell et al. 2009: I05-II3, ici I08).
} 
sation de soi lui permet d'avoir les coudées franches tout en protégeant sa réputation d'enseignant et de pédagogue ${ }^{6}$.

Secret de polichinelle? Dans la Gazette de Lausanne du I2 mars I924, Jean Nicollier tient à signaler aux initiés qu'il n'est pas dupe du stratagème de son confrère :

Excédé d'entendre les champions du latin proclamer sans cesse la supériorité de la culture classique sur l'éducation scientifique, Balthasar pria son vieil ami M. Roorda van Eysinga de railler une peu « les Parvenus de la culture scolaire ». M. Roorda, qui n'a pas fait de latin et qui l'avoue, n'y tenait pas plus que ça, cependant, il céda : «Écoute, mon cher, dit M. Roorda, je veux bien te faire plaisir, mais c'est à la condition que tu ne me lâcheras pas d'une semelle. » Et voilà pourquoi Balthasar Roorda tenta lundi soir à la Maison du Peuple de donner le fouet aux latinistes infatués. La nuit suivante, le latin put dormir en paix, la soirée avait été bonne pour lui.

À lire ce compte rendu sarcastique, l'orateur ne semble pas avoir brillé devant son auditoire lors de sa conférence du IO mars I924 sur un sujet faisant déjà débat à l'époque : l'enseignement du latin, au sujet duquel Nicollier et Roorda continueront à croiser le fer dans la Gazette.

Roorda écrit successivement voire parallèlement dans trois des quatre principaux quotidiens locaux - le quatrième étant le Journal de Genève, concurrent prestigieux de la Gazette de Lausanne au sein des milieux libéraux conservateurs. À l'époque et jusque dans les années I960, la presse romande vit paradoxalement un «âge d'or », pour le dire avec Alain Clavien, la guerre imposant une «soudaine accélération du rythme de l'information » (Clavien 2017 : 97). Auparavant, la plupart des périodiques commentaient les nouvelles étrangères, généralement pillées dans les grands titres français et suisses, avec quelques jours de retard ; désormais, le public, qui suit de près les événements du conflit mondial, dans un contexte helvétique fortement politisé et très divisé, exige d'être renseigné rapidement ${ }^{7}$. Les journaux neutres, dits d'information, comme la Tribune de Lausanne et la Tribune de Genève, s'adaptent aux nouvelles attentes : ils s'abonnent aux agences de presse afin de couvrir l'actualité et triplent leurs ventes et leurs bénéfices. Si la plupart des journaux politiques, dits d'opinion, ne peuvent pas rivaliser et connaissent des difficultés, le Journal de Genève et la Gazette de Lausanne, « qui disposaient avant-guerre déjà d'une solide chronique étrangère contribuant à leur réputation internationale » (IOo), quadruplent et doublent respectivement leurs tirages grâce au lectorat français, que leur francophilie leur rallie largement.

6 Au sujet du pseudonyme de Balthasar, voir Michel Froidevaux, « Les Almanachs de Balthasar» (Enckell et al. 2009: I05).

7 Voir Roger Guignard, « Henri s’en va-t-en-guerre... contre la guerre » (Enckell et al. 2009: 8I-89). 
Que ce soit dans la Tribune de Lausanne, la Gazette de Lausanne ou la Tribune de Genève, Roorda développe au fil des semaines et des années une réflexion sur la presse et les journalistes - entre autres sujets en lien plus ou moins étroit avec l'actualité, dont la guerre et le pacifisme, le féminisme, la pédagogie, l'argent ou le progrès. Avec la distance humoristique dont il est coutumier, il observe tantôt l'abonné s'absorbant distraitement dans son quotidien, tantôt le journaliste courant après l'actualité, dont il interroge le rôle dans la société et la responsabilité envers le public, quand il ne réfléchit pas à sa propre posture de chroniqueur. Précisons que son ton se nuance selon la couleur du journal, d'une part, et selon le contexte de guerre ou de paix d'autre part : les articles sur le pacifisme, par exemple, sont nombreux dans les années I9I7-I9I8 pour se raréfier après l'armistice.

\section{La presse a le public qu'elle mérite - ou l'inverse}

Henri Roorda constate d'emblée que le journal est devenu un moyen privilégié de connaissance et de communication, sans autre concurrence que celle du livre, qui touche toutefois un public plus restreint ; depuis le dernier tiers du XIX ${ }^{\mathrm{e}}$ siècle, la " société tout entière [vit] au rythme de la lecture périodique » (Clavien $2017: 65)$ :

À notre époque, on rencontre partout des gens qui ont beaucoup lu. Mais, comme ils ont tous lu les mêmes livres, et, surtout, les mêmes journaux, les opinions personnelles des uns ressemblent étrangement aux idées personnelles des autres. Et leur conversation finit toujours par devenir assommante («Féminisme », Tribune de Genève, 4 octobre 1924, Roorda 2013:356).

La presse a le public qu'elle mérite - à moins que ce ne soit l'inverse ! Roorda jongle constamment avec ces deux axiomes, accusant alternativement les journalistes et les lecteurs de la médiocrité des quotidiens locaux.

Avec «Il y a des lecteurs trop sévères ", il propose une typologie précise et presque caricaturale des abonnés :

Il y a en a qui demandent au journaliste de leur fournir des faits et des idées. D'autres, dont la vie intellectuelle est plus intense, se plongent au contraire dans la lecture de leur journal pour ne plus penser. Ceux-là comprennent que le seul devoir d'un journal quotidien est de paraître chaque jour. [...] Les uns réclament des vérités premières. [...] D’autres veulent absolument des crimes et des accidents. On doit donc leur en fournir. Il y en a qui tiennent à être renseignés avant tout le monde. Pour satisfaire ces impatients, on a renoncé à faire avec méthode la "critique des sources" et l'on a été obligé d'organiser le service des Rectifications et Démentis (Gazette de Lausanne, 4 mars I920). 
Il dégage avec perspicacité les changements à l'œuvre conjointement dans la presse et dans la société : les journaux «de faits et d'idées » sont menacés par les journaux « de crimes et d'accidents » qui s'imposent progressivement grâce à leur stratégie éditoriale et commerciale et à la force de leurs tirages.

Malgré leurs divergences, les feuilles politiques et les titres populaires tendent dès la fin de la guerre à une uniformisation des contenus «faute de beaux sujets de composition ", notamment dans la rubrique toujours très prisée des « Dernières nouvelles de la nuit » :

«[L]es dernières nouvelles » sont les mêmes dans tous les journaux. Je ne reproche pas aux journalistes de manquer d'imagination. Mais ils ont le tort de payer des sommes considérables à une Agence télégraphique centrale, laquelle, pour ne pas se fatiguer, envoie les mêmes dépêches à tous ses clients ("La mévente du journal », Gazette de Lausanne, 22 septembre I92I, Roorda 202I : I2O).

Le recours systématique aux agences de presse, qui répercutent depuis le retour de la paix une actualité moins brûlante que pendant le conflit, émousse la curiosité des lecteurs ; désappointés, ils se rabattent sur un seul journal, avec pour conséquence la chute des ventes, en déduit le chroniqueur.

"Quand on écrit pour soutenir une cause, on devrait constamment rappeler au lecteur que la thèse contraire est juste aussi » (« Promenons-nous le dimanche », Tribune de Lausanne, 22 juillet 1917, Roorda 2013 : 26). À la presse politique traditionnelle, représentée par la Gazette de Lausanne qui l'accueille pendant sept ans, Roorda reproche un traitement de l'information unilatéral et partisan, nuisant à la formation des opinions et empêchant le débat démocratique :

Le titre que j’ai donné au présent article ne doit pas inquiéter les abonnés de la Gazette de Lausanne. Fidèle à ses principes, ce journal continuera à répudier énergiquement les doctrines des internationalistes. En parlant de «notre internationalisme», je me contenterai d'énoncer quelques-unes de ces propositions générales que peuvent admettre les plus fermes soutiens de la société dans laquelle nous avons le bonheur de vivre (« Notre internationalisme », Gazette de Lausanne, I7 janvier 1924, Roorda 2013 : 285).

Le titre en question, « Notre internationalisme», rappelle celui de «Mon internationalisme sentimental ", essai vibrant de ses idéaux libertaires et pacifistes que Roorda a publié en I9I4 dans les Cahiers vaudois, mais avec lequel la chronique de 1924 n'a rien à voir, ironise-t-il : elle porte sur l'usage abusif du terme "internationalisme". 
Quant à la nouvelle presse d'information, à laquelle l'attache sa double collaboration à la Tribune de Lausanne et à la Tribune de Genève, elle n'est pas épargnée non plus. Roorda lui reproche de suivre une logique non pas de formation mais de relais des opinions, dans le but avant tout de gagner des lecteurs - souvent au mépris de la déontologie. Le 25 mars 1920, par exemple, dans une chronique de la Gazette de Lausanne intitulée "Une explication ", il pointe le sensationnalisme au détour de l'explication de l'injonction « $\mathrm{Ne}$ nous frappons pas ", titre général de ses articles hebdomadaires choisi par lui en hommage à un de ses maîtres à penser et à rire :

Le philosophe Alphonse Allais s'en était déjà servi pour intituler l'un de ses principaux ouvrages.

"Se frapper" signifie : s'affecter de terreur ou de crainte (Littré et Beaujean). En répétant: «Ne nous frappons pas!», je conseille à mes contemporains de ne pas se laisser trop violemment émouvoir par les nouvelles sensationnelles que le télégraphe nous apporte chaque jour. (Obligés d'être très concis, les télégraphistes manquent souvent de "nuances".) Plus généralement, c'est aussi se frapper que d'associer à l'Événement une importance excessive (Roorda 2013: 92).

L'allusion au télégraphe, dont l'invention au milieu du XIX siècle a rendu possible la transmission immédiate de l'information, est révélatrice sous la plume de Roorda, qui en fait le symbole de la presse moderne.

Cinq ans plus tard, le 24 octobre 1924, Roorda s'interroge, avec "Une bonne nouvelle », sur l'opportunisme des journalistes qui répondent cyniquement au goût, selon lui avéré, des lecteurs pour les faits divers : « Rien ne prouve que les choses aillent plus mal qu'autrefois ; mais ceux qui lisent les journaux veulent des histoires dramatiques; et quand il y a pénurie de crimes et d'accidents, le journaliste est obligé d'en inventer » (Roorda 2013:363).

Il n'est pas anodin que cette chronique ait paru dans la Tribune de Genève où sont proposés, " à côté de quelques articles d'information [...], essentiellement des faits divers, ainsi que deux romans feuilletons destinés au public féminin » (Clavien 2017: 72). Roorda prend pour cible large le quotidien qui l'emploie et s'empare subrepticement du contre-pouvoir que peut abriter l'espace de la rubrique. Ainsi que l'observe Marie-Ėve Thérenty, qui identifie cette stratégie chez divers journalistes dans la presse française dès le tournant du xxe siècle, « la chronique, en apparence discours de la marge et de la virtuosité littéraire, peut jouer de cette position excentrée [...] pour déconstruire la machine journalistique, son écriture, son idéologie, pour prendre le contrepied de l'industrie du journal» (Thérenty 2007 : 243-244).

Dans la Gazette de Lausanne du 4 décembre 1924, Roorda revient à la charge sur la manipulation et la dramatisation de l'information : 
Si l'on s'en tenait aux nouvelles que les feuilles publiques lancent chaque matin dans la circulation, on devrait croire que le monde est dans un état d'effroyable désordre et que l'humanité va bientôt sombrer dans la démence. Le journaliste ne nous parle des trains que lorsqu'ils déraillent. Il ne s'intéresse qu'aux piétons qui se font écraser par des automobiles. Contre les autres (qui sont pourtant plus intelligents), il fait la conspiration du silence. C'est seulement lorsqu'un village est détruit par un incendie ou par une avalanche qu'on nous révèle son existence (« Le Permanent », Roorda 2013 : 370).

Pourfendant le catastrophisme mensonger de ses confrères, il décrit avec conviction « l'ordre admirable » de l'univers et le bon fonctionnement de la société, avant de conclure prosaïquement : "En dépit de tout le mal qu'on dit des C.F.F., je certifie que mon train n'a jamais déraillé. Il m'a toujours déposé à l'endroit précis où je voulais me rendre »(370-37I).

Écœuré autant par le traitement malhonnête de l'information dans la presse que par la complaisance coupable des lecteurs, Roorda rêve du lancement d'un hebdomadaire à contre-courant, dont le titre déjà est emblématique. Apolitique et détaché de l'actualité, Le Permanent privilégierait «l'essentiel » à la place de l'accidentel, « tout ce qui est bon et durable » à la place de l'instantané, et ferait la part belle à la poésie et à la philosophie plutôt qu'aux faits divers :

Le journal nouveau que j'attends [...] leur dirait [à mes contemporains inquiets] que la terre continue à tourner autour de son axe d'un mouvement rigoureusement uniforme. Et, pour les hommes, cela est beaucoup plus important que la démission possible de M. Mussolini (37I).

Tout aussi idéaliste est le « Journal blanc », pour reprendre le titre de sa chronique, dont Roorda attribue l'idée à un confrère probablement imaginaire : pour lutter contre le "remplissage" de la presse quotidienne, Ludovic Martial, directeur de L'Indépendant, conçoit un quotidien inédit dont " une partie de chaque page plus ou moins grande resterait en blanc lorsque la "matière" serait peu abondante " (Roorda 2013 : 437). Le changement de régime, cependant, suscite le mécontentement des acheteurs au numéro qui exigent d'être remboursés. La morale de la fable se retourne contre leur manque de discernement qui confine à la bêtise : " Pour la plupart des lecteurs, les nouvelles deviennent intéressantes par le simple fait que le journal les publie » (438).

Plus utopique encore est son projet de remanier L'Illustration : exaspéré par les commentaires ineptes sur « l'actualité politique » que l'hebdomadaire, bien réel celui-ci, ressasse, Roorda en vient à souhaiter qu'il soit publié « sans phrases, un beau journal illustré qui nous montrerait des choses dont il ne nous dirait pas le nom. [...] Et nous nous laisserions émerveiller 
par la richesse de l'univers (" Journaux illustrés ", Gazette de Lausanne, I6 août 1923, Roorda 20I3:236).

\section{Une petite leçon de savoir-lire}

Enhardi par son succès dans les quotidiens vaudois et genevois, Roorda se décide à publier septante-cinq chroniques extraites principalement de la Tribune de Lausanne dans À prendre ou à laisser (1919) et quarante et une extraites de la Gazette de Lausanne dans Le Roseau pensotant (1923). Leur présentation en recueil les soustrait les unes et les autres à la péremption de l'actualité et transforme significativement leur statut générique et leur réception - l'auteur ne manque pas de s'en inquiéter dans sa préface d'À prendre ou à laisser :

B.- [...] Mais j'ai encore quelques scrupules. Il y aura dans mon livre beaucoup de passages bien quelconques, bien médiocres ; ne devrais-je pas d'abord les améliorer?

Moi. - Non. Cela exigerait beaucoup de temps. Et puis, si tu commençais à corriger tes phrases, tu ne t'arrêterais jamais. Il faut, d'ailleurs, qu'il y ait dans un livre des longueurs et du remplissage. Cela permet au lecteur de souffler (Roorda 2012:8).

Le subterfuge du pseudonyme se révèle particulièrement propice à l'introspection critique : dans ce dialogue paradoxal à une seule voix, Roorda encourage son vieil ami Balthasar à publier, malgré les doutes qui le taraudent, son « recueil d'articles qui ont déjà paru ici et là » (7).

À relever que la question du support de publication est déterminante : les chroniques de Roorda auraient-elles passé à la postérité si le relais n'avait pas été pris par le biais de recueils en librairie à l'intention d'un lecteur moins distrait ou pressé que l'abonné du journal ?

Bien que ses articles sur la presse et les journalistes soient relativement nombreux dans les quotidiens auxquels il collabore, Roorda n'en sauve qu'un seul de la lecture à court terme et de l'oubli programmé dans À prendre ou à laisser. Publié initialement dans la Tribune de Lausanne du I6 septembre I9I7, «L'aveu du journaliste » est donc à apprécier sur le long terme, comme une synthèse de portée générale et définitive.

Il sagit d'un petit apologue drôle et didactique, dont les majuscules allégorisantes ont pour effet de suspendre la question de la référence - aucun nom de journaliste, aucun titre de quotidien ne sont mentionnés explicitement. Il s'ouvre sur un récit de rêve activant une mise en abyme : 
Lecteur, je veux, aujourd'hui, te dire la vérité. La nuit dernière, dans un songe, j'ai vu venir vers moi mes illustres confrères, les Journalistes, au nombre de 512 839. Ils m'ont dit : « Nous t'avons choisi, toi, infime ver de terre parce que tu t'adresses exclusivement, dans un des quotidiens les plus répandus en Europe, aux lecteurs du dimanche, c'est-à-dire à des gens qui ont le temps de réfléchir. Dans ton prochain article, tu leur diras Notre Secret. Sois sans crainte : tu seras à la hauteur, car nous aurons soin de t'inspirer. Puis j'entendis un bruit formidable : c'étaient ces messieurs qui s'en allaient à travers mon plafond (46).

Multipliant, dans la meilleure tradition rhétorique, les précautions oratoires, Balthasar, à moins que ce ne soit Roorda, passe de l'apostrophe à l'aveu de modestie et à la flatterie hypocrite pour s'attirer la bienveillance et l'attention du lecteur auquel il s'apprête à donner, au nom de ses confrères, une petite leçon de savoir-lire.

Il commence par s'étonner de sa soumission aveugle à l'autorité morale et intellectuelle du journaliste, que ce dernier en use ou pas : «Tu reviens quotidiennement vers moi avec confiance pour que je te dise ce que tu dois penser » (46). Roorda s'applique ensuite à réévaluer les réelles compétences de ses confrères, tout en les dégageant des trop lourdes responsabilités qui leur sont attribuées - sans pour autant décrier la profession : au journaliste appartiennent l'érudition, la logique, l'esprit et l'éloquence ; il est généralement sincère et ne cherche pas à tromper sciemment qui que ce soit même s'il n'hésite pas à inventer « en cas de pénurie » quelques faits divers saisissants ; il est fidèle, utile et distrayant dans bien des circonstances de la vie quotidienne :

Je te fournis des sujets de conversation pour les minutes où tu es avec des gens à qui tu n'as rien à dire. Je t'empêche de penser lorsque de graves préoccupations tourmentent ton esprit. Le rythme de ma prose te berce lorsque tu veux somnoler ou t'endormir. Enfin, tu te procures, avec raison, deux ou trois journaux lorsque tu vas prendre le train (48).

Le chroniqueur ne se fait pas d'illusion quant à la sensibilité et la concentration du lecteur, sur lequel il porte un regard sévère et néanmoins fraternel.

Malgré toutes ses qualités relatives et non absolues, le journaliste n'est pas plus historien que moraliste aux yeux de Roorda. Il ne détient ni ne prétend détenir la vérité : «Devant les graves questions pour lesquelles les hommes se battent, pourquoi en saurais-je plus long que les scieurs de long, les ferblantiers ou les dentistes? » (48). Tenu par des délais serrés et toujours pressé, il n'a guère le temps de la réflexion : il doit fournir des informations, remplir les colonnes du journal, suivre le rythme de la production. 
Roorda termine sa démonstration en critiquant la crédulité, la naïveté et la docilité du lecteur, avant d'en appeler à son esprit critique, à son bon sens et surtout à son libre-arbitre :

Malheureux! - Parce que mes discours sont tirés à 50000 ou 200000 exemplaires et répandus sur tout le pays - simple question de mécanique et d'organisation -, tu en conclus qu'ils contiennent une plus grande part de vérité que tes propres paroles et que celles de tes amis (47).

En fin de compte, n'est-ce pas contre la diffusion de masse, dont il pressent les dangers, que Roorda met en garde le lecteur ? Résultant de la démocratisation et de la marchandisation de la presse, que favorisent la baisse des prix des abonnements, le développement de la vente au numéro et la large diffusion des imprimés, elle se caractérise par la communication de quelques émetteurs à un grand nombre de récepteurs, par l'unilatéralité du message et par l'indifférenciation de l'information.

«L'aveu du journaliste » se termine sur trois conseils à méditer :

Si tu veux de belles pensées, tu les trouveras dans les livres qui ont été composés lentement. Si, avant de prendre parti pour de grandes luttes, tu hésites, consulte ta femme. Et, s'il m'arrive encore par habitude, de te révéler l'avenir, ou de t'indiquer la cause pour laquelle tu dois toujours être prêt à tout sacrifier, songe à mon inévitable ignorance ; souris et sois indulgent pour mon toupet professionnel ! (49)

Roorda aura mis lui-même en pratique le premier en publiant, outre $\grave{A}$ prendre ou à laisser et Le Roseau pensotant, trois autres essais : Avant la grande réforme de l'an 2000 (1924), Le débourrage des crânes est-il possible? (1924) ${ }^{8}$ et Le Rire et les rieurs (1925). Gageons que sa misogynie - qui contribue à donner un ton d'époque à ses chroniques - l'aura dissuadé de suivre le deuxième. Quant au troisième, il n'en aura pas toujours bénéficié, au vu de l'abondant courrier de lecteurs perplexes ou courroucés à lui adressé.

\section{Un plaidoyer pour la diversité}

Comment Roorda compose-t-il pratiquement avec les changements qui transforment la presse en profondeur au tournant $\mathrm{du} \mathrm{xx}^{\mathrm{e}}$ siècle ? Comment exerce-t-il son second métier de journaliste, lui si prompt à brocarder, certes avec indulgence, ses confrères ? Dans la Gazette de Lausanne du 22 mars

8 Dans une des sections de ce petit pamphlet, intitulée "Le journal et le livre ", Roorda reprend succinctement quelques-unes de ses idées sur la presse : l'autorité du journaliste, le remplissage du journal, la manipulation de l'opinion. 
I923, il tient à rappeler qu'il n'est pas habilité à traiter de sujets érudits, « héroïques » ou "dangereux », mais qu'il est " préposé à l'annonciation des vérités éternelles » :

Le jour où Monsieur le directeur de la Gazette de Lausanne a bien voulu me confier les fonctions que je remplis dans ce journal depuis plus de trois ans, il m'a fait comprendre très poliment que les questions difficiles seraient traitées par des gens plus instruits que moi. J'en convins tout de suite : je ne pouvais parler au public que des choses que tout le monde connaît ( $\mathrm{Ma}$ documentation », Roorda 2013: 190).

Avec «Soyons sérieux! » et « Tout augmente», chroniques respectivement du II novembre I9I7 et du 5 mai I9I8 qui ponctuent les premiers mois de sa collaboration régulière à la Gazette de Lausanne, Roorda saisit le prétexte des lettres, apparemment authentiques, d'une lectrice « affligée » et de lecteurs «mécontents ", tous anonymes, pour préciser et justifier sa posture de chroniqueur. Soulignons au passage que Roorda établit la même complicité amicale ou crispée, le même bavardage grave ou enjoué avec ses destinataires, parfois désagréablement incarnés quand ils prennent la plume, qu'avec les interlocuteurs virtuels animant ses billets, dont son ami Philippe malheureux en ménage, son cousin Maxime ou la petite Éléonore " qui ne comprend rien aux mathématiques ». Privilégiant le dialogisme, la chronique se construit sur le modèle de la causerie familière : quand elle ne mime pas l'échange épistolaire, elle semble prolonger une discussion de comptoir, de terrasse de café ou de boulevard - mais jamais de salon ni de bibliothèque. Roorda choisit de confronter son point de vue personnel non pas à la doxa mondaine ou érudite, mais à la doxa populaire, pour en vérifier la pertinence ou au contraire l'indigence.

Les reproches des abonnés, que le chroniqueur relaie avec une contrition à moitié feinte, portent en réalité moins sur son manque de sérieux que sur l'ambiguïté de sa posture énonciative, jugée tantôt d'une " profondeur obscure », tantôt d'une « légèreté presque inconcevable » (« Tout augmente », Tribune de Lausanne, 5 mai I9I8, Roorda 20I3 : 49). Piqué au vif, il imagine sous forme de boutade une pseudo-signalétique textuelle :

Puisque le public est si peu homogène, puisque les goûts sont si divers, je m'engage à placer désormais, tout au long de mes articles, des poteaux indicateurs portant l'un des mots : sérieux-très gai-profond-idiot-ironiquemorale-énorme.

Comme cela, du moins, le lecteur sera averti et il pourra choisir ( Soyons sérieux ! », Tribune de Genève, II novembre 1917, Roorda 2013 : 3I). 
Roorda revendique la plasticité d'un "je" virevoltant, protéiforme et histrionesque, qui donne paradoxalement son unité à la chronique. Dans « Tout augmente », il s'amuse à le démultiplier en trois Balthasar, le premier sérieux et ennuyeux, le deuxième drôle et facétieux, et le troisième servant « à combler le vide qu'il y a entre les deux premiers »:

Je suis toujours le même; mais ce n'est pas toujours le même qui écrit. Nous sommes trois. Je veux dire que Balthasar est un nom générique (comme Pharaon, Bourbon ou Francilion, par exemple).

Le public couché qui, le dimanche matin, attend impatiemment La Tribune, est composé des éléments les plus divers; et il serait impossible à un chroniqueur seul de contenter tout le monde ("Tout augmente », Tribune de Lausanne, 5 mai 1918, Roorda 2013: 49).

Les postures ou plutôt les impostures de Roorda sont ainsi animées par la volonté non pas de plaire au plus grand nombre, mais de répondre à l'hétérogénéité du public ; cette généreuse aspiration, sans danger pour la réputation du journal, en aura sans doute rassuré le directeur - à défaut de convaincre les lecteurs.

En souvenir peut-être de ses débuts difficiles à la Gazette, Roorda inaugure le 2 février 1923 sa collaboration à la Tribune de Genève avec une «Déclaration de principes » à la gravité mi-sincère mi-parodique - comme le prouve ce clin d'œil à Baudelaire : «La responsabilité du journaliste est terrible. Dans mes nuits d'insomnie, je pense souvent aux égards que je dois à mon lecteur, mon frère » (Roorda 2013: I80).

Le chroniqueur ne prétend ni à l'originalité ni à la vérité dont se réclament ses confrères dans leur quête effrénée de renseignements inédits et de faits établis et attestés. Leur opposant la sérénité et la fantaisie, il s'engage à instruire sans endoctriner et à divertir sans agacer, avec toujours le même mot d'ordre à la fois moral et esthétique : «[T]âchons de mettre de la diversité dans notre unité » (I80).

Son plaidoyer pour la diversité, discret mais constant, amène Roorda à réfuter sans le savoir l'accusation, souvent portée contre le chroniqueur, de complicité de classe avec son lecteur. Il peut en effet se targuer de tour à tour séduire et irriter le public aisé et cultivé de la Gazette de Lausanne et celui, plus populaire et moins savant, de la Tribune de Lausanne ou de la Tribune de Genève : "J'ai un pied dans la bourgeoisie éclairée, et un autre dans le prolétariat conscient ", écrit-il en I9I9 dans « Ça ne va pas trop mal » ( $L a$ Tribune de Lausanne, I2 octobre 1919, Roorda 2013: 80).

Le chroniqueur n'aime pas les lecteurs, pour pasticher le titre du célèbre pamphlet de Roorda sur la pédagogie. Il n'aime pas non plus les journalistes. Convaincu que la presse, autant que l'école, est nécessaire et utile mais aussi potentiellement nocive, il tend aux journalistes comme aux pédagogues, 
dont il partage les ambitions et les tribulations, aux lecteurs comme aux écoliers, qui le désolent souvent par leur docilité, un miroir grossissant afin de leur faire prendre conscience de la relativité voire de la fragilité de toute parole proférée à partir d'une position d'autorité.

\section{Bibliographie}

Chambure, Alexis de, À travers la Presse, Paris, Fert Albouy, I9I4.

Clavien, Alain, La Presse romande, Lausanne, Antipodes/Société d'histoire de la Suisse romande, 2017.

Enckell, Marianne et al., Henri Roorda et l'humour zèbre, Lausanne, HumuS/ Association des amis de Henri Roorda, 2009.

Roorda Henri, Le Pessimisme joyeux : 404 pépites d'humour et d'humeur, préf. Michel Froidevaux, Lausanne, HumuS/Association des amis de Henri Roorda, 2009.

-. À prendre ou à laisser : le programme de lecture du professeur d'optimisme, Paris, Mille et Une Nuits, 2012 [1919].

-. Les Saisons indisciplinées, éd. Gilles Losseroy, Doris Jakubec et Carine Corajoud, Paris, Allia, 2013.

-. Ainsi parlait Balthasar, chroniques drolatiques (I92O-I92I), Le Thoronet, La Première Heure, 2020.

-. Intelligence à louer, éd. Jonathan Wenger, Genève, La Baconnière, 202I.

Thérenty, Marie-Ève, La Littérature au quotidien. Poétiques journalistiques au XIX siècle, Paris, Seuil, 2007. 
